\title{
Método de delimitação celular em imagens ER/PR de câncer de mama
}

\author{
João Victor Kenji Tamaki ${ }^{1}$, Johanna Elisabeth Rogalsky ${ }^{1}$, \\ Sergio Ossamu Ioshii ${ }^{2}$, Lucas Ferrari de Oliveira ${ }^{1}$ \\ ${ }^{1}$ Departamento de Informática - Universidade Federal do Paraná (UFPR) \\ Caixa Postal 15.064 - 91.501-970 - Curitiba - PR - Brazil \\ ${ }^{2}$ Pós-Graduação de Tecnologia em Saúde da PUCPR \\ Pontifícia Universidade Católica do Paraná (PUCPR) \\ Curitiba, PR - Brazil \\ jvictortamaki@gmail.com, johanna.elisabeth8@gmail.com \\ sergio.ioshiiepucpr.br, lferrari@inf.ufpr.br
}

\begin{abstract}
This paper describes a method to be used as an aid to pathologists in the early detection/diagnosis of breast cancer in immunohistochemical images. Besides, the treatment prognosis can make use of these results. A developed cell delimitation method uses an image processing techniques that will serve as a basis for the future development of a cell counting technique. The implemented method showed that the segmentation/delimitation stage is efficient for the progress of the work, with no quantitative results.
\end{abstract}

Resumo. Este artigo descreve um método a ser usado como auxílio para patologistas na detecção/diagnóstico precoce de câncer de mama em imagens imunohistoquímicas. Além disso, esses resultados podem ser utilizados para o prognóstico do tratamento. Foi desenvolvido um método de delimitação de células utilizando técnicas de processamento de imagens que servirá de base para o desenvolvimento futuro de uma técnica de contagem celular. A implementação do método mostrou que a etapa de segmentação/delimitação é eficiente para o andamento do trabalho, não possuindo resultados quantitativos.

\section{Introdução}

O câncer de mama é a formação de tumores resultante do processo de crescimento desordenado de células da mama [INCA 2021b]. As células de câncer de mama podem conter certos tipos de receptores, que são proteínas que buscam determinadas substâncias sanguíneas. Ao receberem progesterona (PR) ou estrogênio (ER), estas proteínas contribuem para o crescimento desordenado do câncer que pode invadir órgãos ou tecidos adjacentes, e assim, afetar o funcionamento do corpo [Martin et al. 2019].

O câncer de mama é o câncer que mais acontece em mulheres no mundo todo [Pereira et al. 2019], inclusive no Brasil. Sendo o câncer com maior mortalidade entre mulheres [INCA 2021a], tornando-se um problema de saúde pública no mundo todo [da Silva and Riul 2011] por causa da alta taxa de casos. Sendo assim, existe uma preocupação para diminuir essas estatísticas. Um dos modos de redução desta taxa de 
mortalidade é o diagnóstico e início do tratamento de forma precoce [INCA 2021b]. Estudos feitos pelo Instituto Nacional de Câncer (INCA), mostram que a taxa de sobrevida em cinco anos, nos anos de 2010 até 2014, foi de 75,2\% [Instituto Nacional de Câncer 2019].

Uma das formas de prognóstico são as imagens de imunohistoquímica (IHQ) de tecidos. O teste de IHQ detecta antígenos específicos por meio de anticorpos monoclonais que são marcados por substâncias fluorescentes [Bochtler et al. 2018]. A classificação de células de mama utilizando o teste imunohistoquímico para ER e PR para a identificação e diagnóstico por imagem é um dos métodos utilizados para a confirmação ou não do câncer [Bochtler et al. 2018]. Com esse exame, é possível ver as células que foram coradas significando níveis de câncer.

Porém, é necessário um patologista para analisar a imagem de câncer de mama [Mouelhi et al. 2018]. O grau de experiência do patologista é decisivo na hora de escolher se será feito quimioterapia, radioterapia, cirurgia ou apenas acompanhamento, o que pode ser decisivo para o paciente, assim como, é função do patologista detectar a existência de nódulo ou não e se é benigno ou maligno [Equipe SBP 2016].

Para auxiliar o diagnóstico propomos uma metodologia para a contagem de células que ajudará o patologista a ver as imagens imunohistoquímicas de forma mais rápida para auxiliar na precisão do diagnóstico do exame. Desse modo, este trabalho apresenta uma técnica de delimitação de células receptoras de estrogênio e progesterona para realizar a contagem de células, fazendo uso de técnicas de visão computacional.

\section{Trabalhos Relacionados}

Para o estudo em questão, foi analisado o método de segmentação e classificação de imagens imunohistoquímicas feita por [Mouelhi et al. 2018] como forma de embasar a pesquisa em um método que já apresentou bons resultados. O método se baseia em várias técnicas de Processamento de Imagens aplicadas em imagens de células de câncer de mama para extrair as células e realizar a classificação das mesmas. Das etapas do trabalho original desenvolvemos até a etapa de identificação das células posteriormente, serão desenvolvidas as etapas de quantificação e classificação das células de câncer de mama.

\section{Materiais e Métodos}

\subsection{Imagens}

As imagens utilizadas tem o formato .png e dimensões de 400x300 pixels. Elas são blocos criados a partir de imagens .czi feitas com lentes de aumento 40x a partir de lâminas de biópsia de câncer de mama. Para esse estudo inicial foram utilizadas 10 imagens de 24 pacientes. Alguns exemplos podem ser vistos na Figura 1.

Elas apresentam aspectos variados devido ao fato de representarem pacientes com diagnósticos de câncer de mama em fases diferentes. As colorações marrom e azul identificam, respectivamente, células que reagiram a 3,3'-diaminobenzidina(DAB), mostrando células cancerígenas, e células que reagiram a hematoxilina, mostrando a parte não-tumoral ou porções de tecido tumoral que não reagiram aos receptores alvo (ER/PR). 


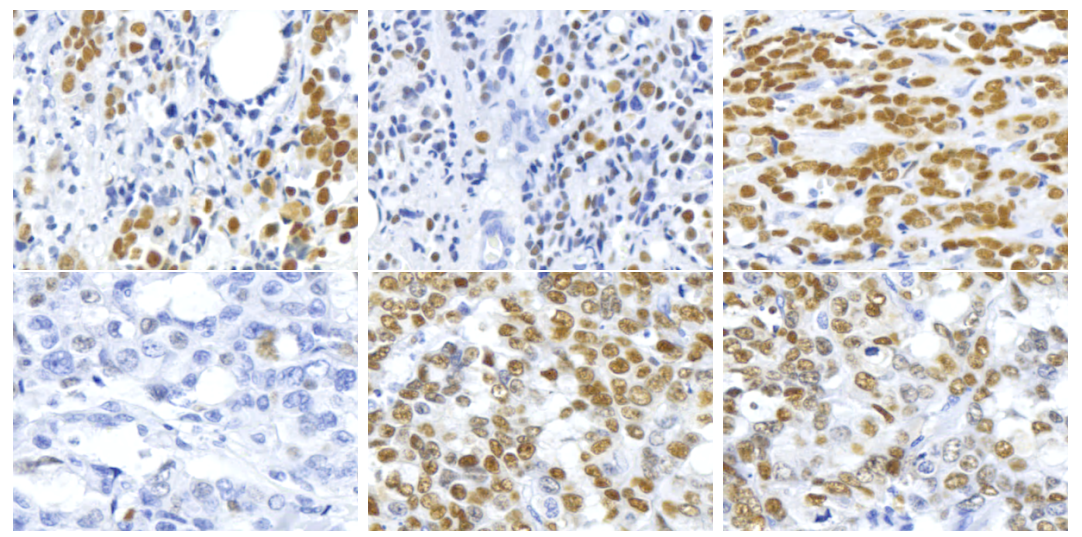

Figura 1. Exemplos de imagens usadas de tecidos de pacientes com câncer de mama em diferentes estágios.

\subsection{Método Proposto}

O método proposto é um contador e classificador de células em imagens de câncer de mama baseado no trabalho de [Mouelhi et al. 2018]. Para isso, é usado um conjunto de imagens apresentado como entrada para realizar as funções de processamento de imagem. Para esse estudo, foi utilizado Python e funções de bibliotecas como o OpenCV, Numpy e o Pandas.

Primeiramente, foi feito o aumento do contraste para conseguir uma imagem mais nítida. No segundo passo, foi realizado um thresholding nas imagens para obter uma imagem binária que destacasse a localização das células para a segmentação. Como preparação para o thresholding, foi feito o agrupamento médio para cada pixel da imagem. Também foi realizada a filtragem Gaussiana para a remoção de ruídos.

Com essa imagem base, aplicamos o método de segmentação composto de 3 etapas: aplicação do filtro laplaciano modificado, dos filtros morfológicos e o algoritmo Watershed. O filtro laplaciano modificado que foi utilizado se baseia em cada pixel da imagem ser igual a $\alpha \cdot(\delta(I T)+\epsilon(I T))-(\max (I T)-I T)$, sendo que o $\alpha$ utilizado é 4, IT é a imagem de entrada do filtro laplaciano e $\delta(I T)+\epsilon(I T)$ é a imagem de dilatação mais a imagem de erosão que é igual a imagem do filtro laplaciano mais o dobro de IT. Os filtros morfológicos consistiram de uma operação de dilatação seguida de fechamento utilizando um elemento estruturante em formato de disco com raio de tamanho 2. Após isso, foi utilizado o algoritmo Watershed.

Por ser um trabalho em andamento e a etapa de contagem de células não estar concluída, não foi possível comparar com a contagem manual de especialistas. Portanto, foram comparados os resultados de duas abordagens de delimitação de células: a primeira usando a imagem base e a segunda usando a imagem após o método de segmentação [Mouelhi et al. 2018]. Como não foi finalizado o aprimoramento do algoritmo de Watershed, foi usada a imagem com o algoritmo padrão. Isso mostra a eficiência do método para delimitar o que é célula ou não, o que mostra que para a etapa de contagem foram detectadas todas as células, mas podendo ainda ter algum ruído. Para uma etapa posterior, será feito a separação de células congruentes no algoritmo aprimorado de Watershed, o que concluíra a implementação do método de contagem de células. 
O método de contagem de células espera-se que elas estejam definidas e bem separadas para efetuar a contagem da delimitação de cada célula. Por meio do sucesso dos métodos de eliminação de ruídos e da divisão das células que serão implementadas, será assegurado que haja uma boa precisão no método e por fim, realizar a contagem e comparar com o resultado de um patologista. No método de [Mouelhi et al. 2018] foi realizada a classificação do núcleo das células e no método proposto será realizada a contagem de células por meio da delimitação das células.

\section{Resultados e Discussão}

As Figuras 2 e 3 apresentam a sequência de imagens resultantes do método desenvolvido até o momento nas imagens de RE e de RP, respectivamente. As imagens aparecem com as células delimitadas, porém ainda são necessários ajustes para aprimorar essa separação celular. Uma etapa já prevista é a retirada do ruído da imagem para conseguir delimitação/segmentação apenas das células. Das 240 imagens testadas nenhuma apresentou resultados em que nenhuma área de tecido tenha sido perdida ou não segmentada. Porém, apresentam ruídos e alguns buracos. Isso mostra que a técnica pode ser utilizada na nossa base de imagens completa que possui 135 pacientes.

Para as próximas etapas e considerando o sucesso da delimitação de cada célula, será possível realizar a contagem pelo método proposto que consiste na contagem de contornos individuais de cada célula. Como mostrado (Figuras 2 e 3), a delimitação das células está em bom estado para prosseguir com os próximos passos. Por enquanto, não é possível ter uma contagem precisa de células, já que apenas foi limitado o espaço no tecido que possui ou não células e não cada célula individualmente. Para todas as imagens utilizadas foi delimitado todo o espaço do tecido, sendo essa delimitação avaliada a princípio pelos pesquisadores, porém para etapas futuras um especialista deverá contar a quantidade de células por imagem.

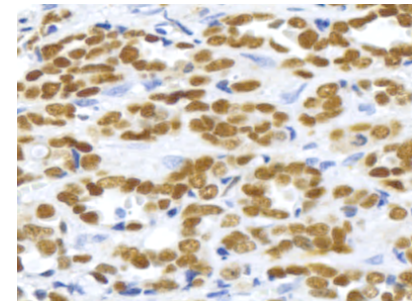

(a)

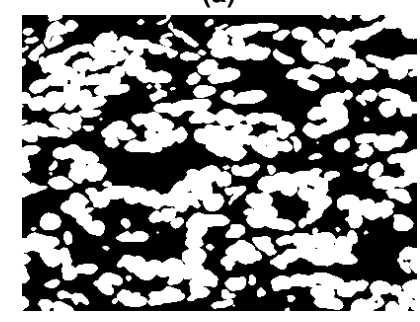

(d)

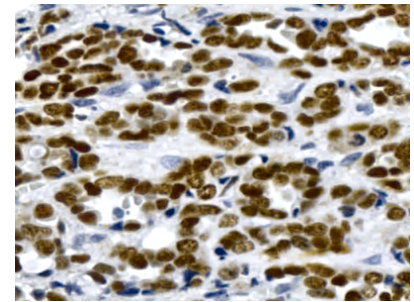

(b)

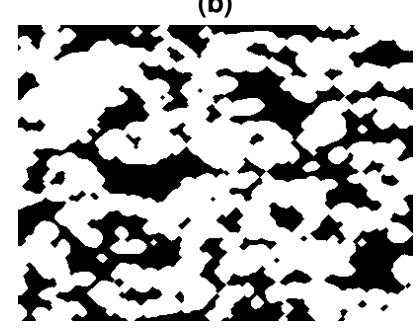

(e)

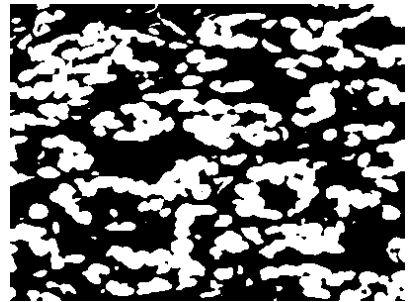

(c)

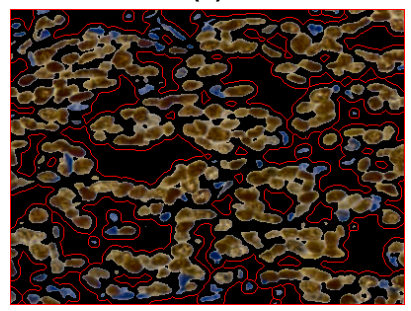

(f)

Figura 2. 2a Imagem RE original. $2 b$ Imagem adquirida ao realizar o aumento do contraste de forma limitada e adaptativa. 2c Imagem adquirida ao realizar o thresholding. $2 \mathrm{~d}$ Imagem adquirida ao realizar o Filtro Laplaciano modificado. 2e Imagem adquirida ao realizar os Filtros Morfológicos. 2f Imagem adquirida ao realiza o algoritmo Watershed. 


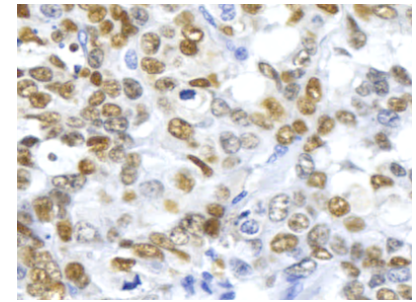

(a)

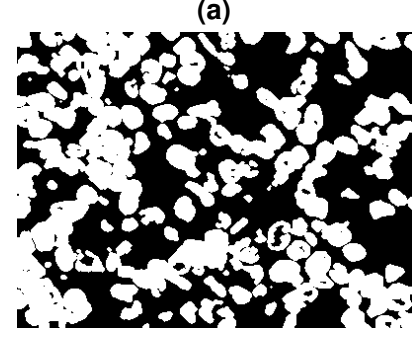

(d)

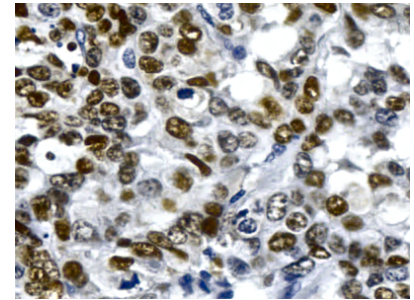

(b)

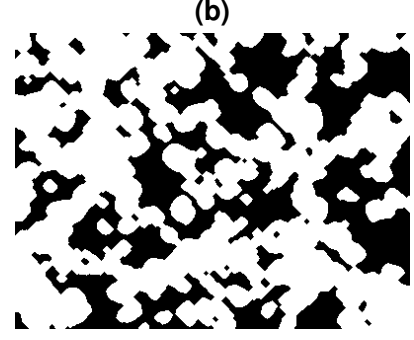

(e)

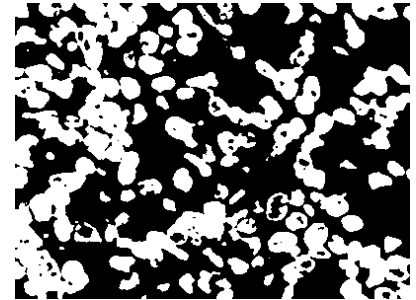

(c)

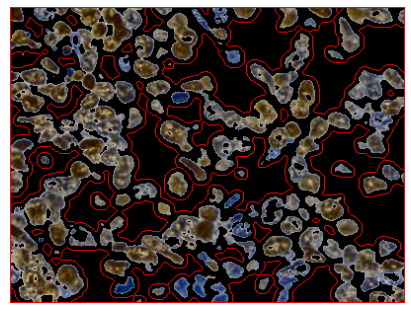

(f)

Figura 3. 3a Imagem RP original. 3b Imagem adquirida ao realizar o aumento do contraste de forma limitada e adaptativa. $3 \mathrm{c}$ Imagem adquirida ao realizar o thresholding. 3d Imagem adquirida ao realizar o Filtro Laplaciano modificado. 3e Imagem adquirida ao realizar os Filtros Morfológicos. 3f Imagem adquirida ao realiza o algoritmo Watershed.

\section{Conclusão}

Este trabalho é a etapa inicial de uma técnica para contagem de células em imagens histopatologicas para o auxílio no diagnóstico de câncer de mama. Para isso, foi usado um método de segmentação para delimitar as regiões dos tecidos das células, visando delimitar as áreas que possuem células e posteriormente separar as células congruentes e realizar a contagem de forma precisa. Os resultados qualitativos obtidos até o momento são promissores e indicam que será possível continuar com a ideia proposta para a contagem de células, pois em nenhuma das 240 imagens tivemos problema de segmentação.

\section{Referências}

Bochtler, T., Löffler, H., and Krämer, A. (2018). Diagnosis and management of metastatic neoplasms with unknown primary. Seminars in Diagnostic Pathology, 35(3):199-206.

da Silva, P. A. and Riul, S. d. S. (2011). [Breast cancer: risk factors and early detection]. Revista brasileira de enfermagem, 64(6):1016-1021.

Equipe SBP (2016). O papel do patologista na luta contra o câncer.

INCA (2021a). Causas e Prevenção: Estatísticas de câncer.

INCA (2021b). Tipos de câncer: Câncer de mama.

Instituto Nacional de Câncer (2019). Breast cancer in brazil: synthesis of information. https://www.inca.gov.br/sites/ufu.sti.inca.local/ files/media/document//a_situacao_ca_mama_brasil_2019.pdf. Accessed: 2019-10-23.

Martin, V., Valera, A., De Joffrey, M., Banfi, S., and Mazzucchelli, L. (2019). Implementation of the 2018 Human Epidermal growth factor Receptor 2 guideline by Ameri- 
can Society of Clinical Oncology/College of American pathologists will reduce falsepositive tests. Archives of Pathology and Laboratory Medicine, 143(4):411-412.

Mouelhi, A., Rmili, H., Ali, J. B., Sayadi, M., Doghri, R., and Mrad, K. (2018). Fast unsupervised nuclear segmentation and classification scheme for automatic allred cancer scoring in immunohistochemical breast tissue images. Computer methods and programs in biomedicine, 165:37-51.

Pereira, H. F. B. d. E. S. A., Viapiana, P. d. S., and Silva, K. L. T. (2019). Aspectos Clínicos e Patológicos do Câncer de Mama em Mulheres Jovens Atendidas na FCecon entre 2003 e 2013. Revista Brasileira de Cancerologia, 63(2):103-109. 